\title{
DECAY OF SOLUTIONS OF SYSTEMS OF HYPERBOLIC CONSERVATION LAWS
}

\author{
BY J. GLIMM ${ }^{1}$ AND P. D. LAX ${ }^{2}$ \\ Communicated by E. Isaacson, August 8, 1966
}

We are dealing with a system of two partial differential equations of the form

$$
\begin{aligned}
& u_{t}+f_{x}=0, \\
& v_{t}+g_{x}=0 .
\end{aligned}
$$

$f$ and $g$ are functions of $u$ and $v$ subject to the following conditions:

(a) The system (1) is hyperbolic.

(b) The system (1) is genuinely nonlinear in the sense of [2].

(c) The interaction of two shocks of the same family always produces a shock of the same family plus a rarefaction wave of the opposite family.

Denote by $\epsilon$ the oscillation of the initial data. The initial data need not have bounded total variation. Our results are as follows:

THEOREM 1. For epilson small enough the solution of the initial value problem exists for all positive time.

THEOREM 2. If the initial data are periodic the solution decays like $1 / t$.

THEOREM 3. If the initial data are constant outside of a compact interval, the solution decays like $1 /(t)^{1 / 2}$.

All the proofs are based on methods introduced in [1] supplemented by further inequalities which take into account the interaction of shock waves and rarefaction waves of the same family. The new estimates prove that such interactions must occur and that when they do occur they lead to decay in the solution.

\section{BIBLIOGRAPHY}

1. J. Glimm, Solutions in the large for nonlinear hyperbolic systems of equations, Comm. Pure Appl. Math. 18 (1965), 697-715.

2. P. D. Lax, Hyperbolic systems of conservation lawes. II, Comm. Pure Appl. Math. 10 (1957) 537-556.

\section{Massachusetts Institute of Technology and \\ The Courant Institute of Mathematical Sciences, NEW YoRK UNIVERSITY}

${ }^{1}$ Supported in part by the National Science Foundation grant GP-4364.

2 The work presented in this paper is supported by the AEC Computing and Applied Mathematics Center, under Contract AT(30-1)-1480 with the U. S. Atomic Energy Commission. 